\title{
QuantiFERON-TB Gold PLUS versus QuantiFER- ON-TB Gold In-Tube test for diagnosing tuberculosis infection
}

\author{
Soo Han Kim, Kyung-Wook Jo, and Tae Sun Shim
}

Department of Pulmonary and Critical Care Medicine, Asan Medical Center, University of Ulsan College of Medicine, Seoul, Korea

Received: January 2, 2019

Revised : March 6, 2019

Accepted: March 11, 2019

\section{Correspondence to}

Tae Sun Shim, M.D.

Department of Pulmonary and Critical Care Medicine, Asan Medical Center, University of Ulsan College of Medicine, 88 Olympic-ro 43-gil, Songpa-gu, Seoul 05505, Korea

Tel: +82-2-3010-3892

Fax: +82-2-3010-6968

E-mail:shimts@amc.seoul.kr https://orcid.org/0000-00016653-816X
Background/Aims: QuantiFERON-TB Gold PLUS (QFT-PLUS) was developed as a new version of the interferon- $\gamma($ IFN- $\gamma$ ) release assay that contains an extra antigen tube to elicit a CD8+ T-cell response in addition to a CD4+ T-cell response. This study aimed to evaluate the performances of QFT-PLUS versus QuantiFERON-TB Gold In-Tube (QFT-GIT) for detecting tuberculosis (TB) infection.

Methods: Between October, 2016 and May, 2018, 137 participants were prospectively recruited and subjected to QFT-GIT and QFT-PLUS testing. The concordance between tests and performance based on different immune states and/or TB infection risk were evaluated.

Results: The 137 participants were classified as follows: active TB ( $=14)$, TB contact $(n=14)$, screening before biologic therapy $(n=85)$ and other disease $(n=24)$. The positive results for either test were 100\% $(n=14 / 14), 42.9 \%(n=6 / 14), 15.3 \%$ ( $n$ $=13 / 85)$, and $62.5 \%(n=15 / 24)$ in each four groups, respectively. The QFT-GIT and QFT-PLUS test results showed good concordance with $91.2 \%$ agreement and a Cohen's $\kappa$ of 0.807 . The good concordance between two tests was also observed in 64 immunocompromised subjects (agreement of $90.6 \%$ and a Cohen's $\kappa$ of 0.711 ). The intra-class correlation coefficient for each antigen tube of the QFT-PLUS showed a good correlation with the IFN- $\gamma$ release of the QFT-GIT $(\mathrm{TB} 1=0.912, p<0.001$; $\mathrm{TB} 2=0.918, p<0.001)$.

Conclusions: QFT-PLUS showed highly comparable results to those of QFT-GIT for diagnosing TB infection in South Korea as well as in immunocompromised subjects.

Keywords: Latent tuberculosis; Interferon-gamma release tests; CD8-positive T-lymphocyte

\section{INTRODUCTION}

Tuberculosis (TB) is a major global health concern, with an estimated 10.4 million incident cases worldwide in 2016 [1]. Moreover, it has been suggested that approximately one-third of the world's population is latently infected with Mycobacterium tuberculosis (MTb) [2]. The estimated lifetime risk of developing active T'B in subjects with latent TB infection (LTBI) is $5 \%$ to $15 \%$, and the risk is highest in the first 2 years following infection [3]. The early detection and treatment of LTBI in countries with a low or intermediate TB prevalence is an important factor for preventing the progression to active T'B worldwide $[4,5]$. 
After excluding active TB on a chest X-ray (CXR), the diagnosis of LTBI is indirectly made by evaluating the immune response against MTb using a tuberculin skin test (TST) and/or interferon- $\gamma$ (IFN- $\gamma$ ) release assays (IGRA) [6]. The QuantiFERON-TB Gold In-Tube (QFTGIT, Qiagen, Hilden, Germany) and the T-SPOT.TB (Oxford Immunotec, Abingdon, UK) are the two main commercially available IGRA products. Although IGRA products don't have cross-reactivity with the bacillus calmette-guerin (BCG) vaccination like TST, they have cross-reactivity with some other mycobacteria (e.g., $\mathrm{My}$ cobacterium kansasii, Mycobacterium marinum, and Mycobacterium szulgai) [7]. Moreover, IGRA products have a reduced sensitivity in immunocompromised patients and children $[8,9]$. They are also unable to distinguish between recent and remote infections [10].

Several studies have revealed that CD8+ T-cells play an important role in the host defense against MTb by the cytotoxic pathway and the cytosolic pathway [1115]. One study reported that active TB patients with smear-positive sputum showed higher detectable CD8+ T-cell responses than those with smear-negative sputum and LTBI patients, while pulmonary TB patients also showed higher responses than extrapulmonary TB patients [16]. Furthermore, active TB patients with intermediate/high radiologic severity or microbiological TB showed higher detectable CD8+ T-cell responses than those with low radiologic severity or clinical TB [17]. The latter two studies indicated that MTb load is positively correlated with CD8+ T-cell responses.

As previous studies provided good evidence for the role of CD8+ T cell responses in MTb infection, the QuantiFERON-TB Gold PLUS (QFT-PLUS) has been introduced as a new-generation QFT-GIT. The QFT-PLUS contains two antigen tubes, TB1 and TB2: the TB1 tube contains ESAT-6- and CFP-10-derived peptides (TB 7.7 was removed) and is designed to induce $\mathrm{CD}_{4}+\mathrm{T}$ cells response; the TB2 contains both the same long peptides of TB1 and newly designed peptides that stimulate IFN- $\gamma$ production by both $\mathrm{CD}_{4}+$ and CD8+ T cells [18]. Recent studies have reported similar accuracies of QFT-PLUS and with QFT-GIT [17,19-22], and some showed higher CD8+ T cell responses to recent Mtb exposure compared to remote exposure in contact screening [19,22].

This study aimed to evaluate the performance of the new QFT-PLUS assay in diagnosing patients with TB infection to identify whether it can replace the QFTGIT in South Korea, where the incidence of active TB is intermediate (77 per 100,000 in 2016) and the BCG vaccination is mandatory [23].

\section{METHODS}

\section{Study population}

Between October 2016 and May 2018, 182 participants of age $>18$ years were prospectively recruited for diagnosing TB infection from Asan Medical Center, a tertiary referral center in Seoul, South Korea. Most of these cases comprised suspected active TB, recent TB contact, or candidates for TB infection tests. The QFT-GIT and QFT-PLUS assays were performed for each participant. Forty-one participants were excluded owing to not wanting to participate, one owing to an insufficient blood sample for analysis, and three owing to a diagnosis of nontuberculous mycobacterial (NTM) disease showing cross-reactivity with MTb in IGRA (Mycobacterium kansasii, two cases; Mycobacterium marinum infection, one case) [7]. Therefore, 137 patients were involved in the final analysis.

A full history was taken that included age, sex, body mass index, concomitant disease, BCG scar, previous TB treatment, and immune status. CXR, QFT-GIT, and QFT-PLUS were performed for all participants at the baseline examination. CXR findings suggestive of healed TB were identified in the presence of apical fibronodular lesions, calcified lymph nodes, calcified solitary nodules, and pleural thickening [24]. Immunocompromised status was defined as a history of diabetes mellitus, chronic kidney disease, malignancy, organ transplantation, human immunodeficiency virus (HIV), and the use of immunosuppressive agents or steroids (prednisolone $15 \mathrm{mg} /$ day for $>1$ month) [22]. All participants were classified based on TB infection risk (active TB, recent T'B contact, LTBI screening before biologic therapy, or other disease). Active TB group consisted of patients with culture-positive pulmonary TB alone who were enrolled before initiating anti-TB medication, except for one patient who was enrolled after 1 month of anti-TB treatment. Recent TB contact group consisted of patients with household contacts or other close contacts with those with infectious TB. Biologic therapy 
group consisted of patients with immune-mediated inflammatory diseases who were also candidates for biologic therapy such as anti-TNF agents. Other disease group consisted of participants who were finally diagnosed with disease other than TB. Our study protocol was approved by the Institutional Review Board of Asan Medical Center (IRB No.: 2017-0004). All participants provided written informed consent.

\section{QFT-GIT and QFT-PLUS diagnostics}

Peripheral blood sampling and sample processing for both QFT-GIT and QFT-PLUS were performed and interpreted according to the manufacturer's instruction [18]. Peripheral blood was withdrawn simultaneously, directly into test tubes for use in both tests. The tubes were incubated at $37^{\circ} \mathrm{C} \pm 1^{\circ} \mathrm{C}$ for 16 to 24 hours. All active TB patients were enrolled before initiating anti-TB treatment except for one patient who was enrolled after 1 month of anti-TB treatment. The test was recorded as positive if the antigen response (T'B for QFT-GIT, TB1 and/or TB2 for QFT-plus) was $\geq 0.35 \mathrm{IU} / \mathrm{mL}$ above the negative control and $\geq 25 \%$ of the negative control. The results for both tests were recorded as negative if the antigen response minus the negative control was $<0.35 \mathrm{IU} /$ $\mathrm{mL}$ or $<25 \%$ of the negative control with a positive control (mitogen tube) of $\geq 0.5 \mathrm{IU} / \mathrm{mL}$. The result for both tests was recorded indeterminate if the antigen tube minus the negative control was $<0.35 \mathrm{IU} / \mathrm{mL}$ or $<25 \%$ of the negative control with a positive control of $<0.5 \mathrm{IU} / \mathrm{mL}$ or the antigen response of the negative control was $>8$ $\mathrm{IU} / \mathrm{mL}[18]$.

\section{Statistical analysis}

The statistical analyses were performed using the IBM SPSS version 21.0 (IBM Co., Armonk, NY, USA). Continuous variables are expressed as mean \pm standard deviation (SD) or as median and interquartile range (IQR), while categorical variables are expressed as number and percentage. The chi-square or linear by linear associates test was used for comparison of categorical variables. The Wilcoxon signed rank test was used for inter-test comparison of continuous variables. The Mann-Whitney $U$ test or Kruskall-Wallis test was also used for inter-group comparison of continuous variables.

The agreement between QFT-GIT and QFT-PLUS findings was evaluated by calculating the overall per- centage of concordant results and Cohen's $\kappa$ coefficient. The agreement of IFN- $\gamma$ release between the QFT-GIT and QFT-PLUS was evaluated by calculation of the intraclass correlation coefficient (ICC). Linear regression analyses were performed to assess the correlation in IFN- $\gamma$ release between the tests.

The difference between $\mathrm{T} B 2$ and $\mathrm{T} B 1$ antigen responses greater than the cutoff of $0.6 \mathrm{IU} / \mathrm{mL}$ was investigated to evaluate whether IFN- $\gamma$ release of CD8+ T-cells is related to recent TB exposure based on a previous journal by Metcalfe et al. [25].

\section{RESULTS}

\section{Characteristics of study population}

The demographic characteristics of the 137 participants are shown in Table 1. They had a mean \pm SD age of 46.8 \pm 16.3. Immune-mediated inflammatory diseases (IMIDs) were the most common comorbid condition ( $\mathrm{n}=$ $85,62.0 \%$ ). No subjects had chronic kidney disease or HIV infection. Healed TB was seen on CXR in 11 subjects (8.0\%) without evidence of active TB and 10 subjects $(7.3 \%)$ with a previous history of anti-TB treatment. A total of 111 subjects had BCG scarring (81.0\%), while 64 subjects were immunocompromised (46.7\%). LTBI screening before biologic therapy was the most frequent TB infection risk group ( $\mathrm{n}=85,62.0 \%)$, followed by other diseases $(n=24,17.5 \%)$ and active TB $(n=14,10.2 \%)$ or TB contact $(n=14,10.2 \%)$. Other disease group consisted of nine participants with healed TB on the CXR, seven with non-specific CXR abnormality, five with NTM diseases, two with pneumonia, and one under health checkup. The positive rates in each group are shown in Fig. 1.

\section{Comparison of QFT-GIT and QFT-PLUS}

The QFT-PLUS results were comparable to those of QFT-GIT, which resulted in an agreement of 91.2\% and a Cohen's $\kappa$ of 0.807 (Table 2). QFT-GIT showed a 90.5\% agreement and Cohen's $\kappa$ of 0.790 to TB1 and a 92.0\% agreement and Cohen's $\kappa$ of 0.820 to TB2. There were no significant differences in agreement between the QFT-GIT and QFT-PLUS for the different immune states $(p=0.811)$ and no significant trend for TB infection risk $(p=0.558)$.

The median IFN- $\gamma$ release of TB $(5.85 \mathrm{IU} / \mathrm{mL} ; \mathrm{IQR}$, 
2.05 to 10.00$)$ was significantly higher than that of T'B1 (2.62 IU/mL; IQR, 1.06 to 7.91; $p=0.001$ ) and TB2 (3.15 IU/ $\mathrm{mL} ; \mathrm{IQR}, 1.08$ to $8.30 ; p=0.004)$ in the positive QFT-GIT and QFT-PLUS results $(n=40)$. There were significant differences in the median IFN- $\gamma$ release of TB (0.01 IU/ $\mathrm{mL}$; IQR, o to 0.05 ) compared to that of TB1 (0.01 IU/ $\mathrm{mL} ; \mathrm{IQR},-0.01$ to $0.03 ; p=0.003)$ and TB2 (0.01 IU/mL;

Table 1. Baseline clinical characteristics of 137 subjects

\begin{tabular}{|c|c|}
\hline Characteristic & Value \\
\hline Age, yr & $46.8 \pm 16.3$ \\
\hline Sex, male & $67(48.9)$ \\
\hline Body mass index, $\mathrm{kg} / \mathrm{m}^{2}$ & $23.1 \pm 3.6$ \\
\hline \multicolumn{2}{|l|}{ Comorbid conditions } \\
\hline IMIDs & $85(62.0)$ \\
\hline Diabetes mellitus & $6(4 \cdot 4)$ \\
\hline Malignancy & $6(4 \cdot 4)$ \\
\hline Organ transplant recipient & $2(1.5)$ \\
\hline Previous history of anti-TB treatment & $10(7 \cdot 3)$ \\
\hline Positive BCG scarring & $111(81.0)$ \\
\hline Immunocompromised $^{\mathrm{a}}$ & $64(46.7)$ \\
\hline \multicolumn{2}{|l|}{ TB infection risk group } \\
\hline Active TB & $14(10.2)$ \\
\hline Recent TB contact & $14(10.2)$ \\
\hline LTBI screening before biologic therapy & $85(62.0)$ \\
\hline Other diseases & $24(17 \cdot 5)$ \\
\hline
\end{tabular}

Values are presented as mean \pm SD or number (\%).

IMID, immune-mediated inflammatory disease; TB, tuberculosis; BCG, bacillus calmette-guérin; LTBI, latent tuberculosis infection.

${ }^{\mathrm{a}}$ Causes of immunosuppression included diabetes mellitus, chronic kidney disease, malignancy, organ transplantation, human immunodeficiency virus, immunosuppressive medications or steroids (prednisolone $15 \mathrm{mg}$ /day for $>1$ month).
IQR, -0.01 to $0.02 ; p=0.002)$ in the negative QFT-GIT and QFT-PLUS results $(\mathrm{n}=85)$. The ICC showed good correlation between the QFT-GIT and QFT-PLUS (TB1: ICC $=0.912, p<0.001$; TB2: ICC $=0.918, p<0.001)$. In addition, linear regression analyses showed good correlation between tests (TB1: $r^{2}=0.722$; TB2: $r^{2}=0.734$ ) (Fig. $2 \mathrm{~A}$ and $2 \mathrm{~B})$.

Discordant results were found in 12 subjects (Table 3), but a statistical analysis could not be performed due to the small number of subjects. Among them, IFN- $\gamma$ release was in the borderline range of 0.25 to $0.8 \mathrm{IU} / \mathrm{mL}$ in 8 QFT-GIT samples [25].

\section{Comparison of QFT-GIT and QFT-PLUS in immuno- compromised subjects}

The QFT-PLUS results were compatible with those of QFT-GIT in the 64 immunocompromised subjects, which resulted in an agreement of $90.6 \%$ and a Cohen's

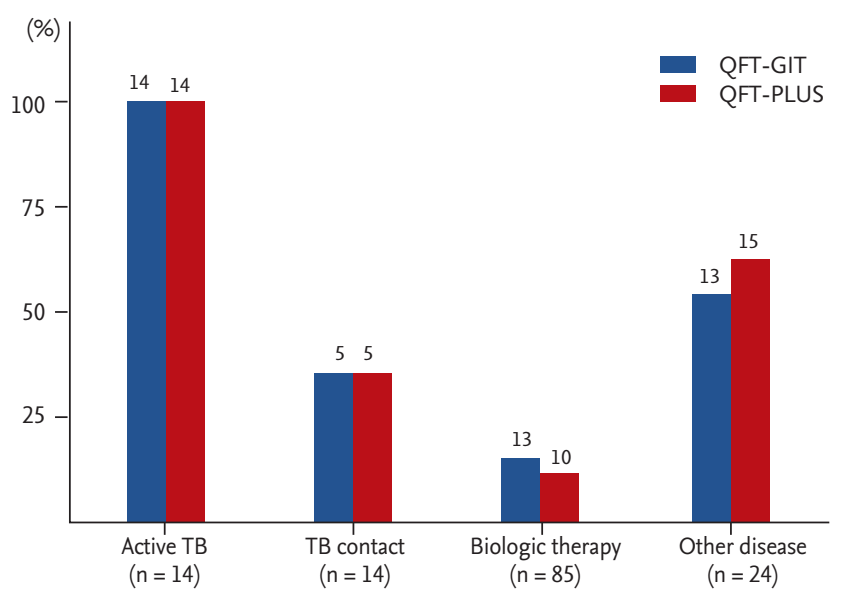

Figure 1. Positive rates of QuantiFERON-TB Gold In-Tube (QFT-GIT) and QuantiFERON-TB Gold Plus (QFT-PLUS) by tuberculosis (TB) infection risk group $(n=137)$.

Table 2. Comparison of QFT-GIT versus QFT-PLUS in 137 subjects

\begin{tabular}{|c|c|c|c|c|c|}
\hline \multirow{3}{*}{ QFT-GIT results } & \multirow{3}{*}{ No. of subjects } & \multicolumn{4}{|c|}{ QFT-PLUS results } \\
\hline & & \multicolumn{3}{|c|}{ Positive } & \multirow{2}{*}{ Negative } \\
\hline & & TB1-nil or TB2-nil & TB1-nil & TB2-nil & \\
\hline Negative & 89 & 4 & 4 & 2 & 85 \\
\hline Positive & 45 & 40 & 39 & 39 & 5 \\
\hline Indeterminate & 3 & 0 & 0 & 0 & 3 \\
\hline Total & 137 & 44 & 43 & 41 & 93 \\
\hline
\end{tabular}

QFT-GIT, QuantiFERON-TB Gold In-Tube; QFT-PLUS, QuantiFERON-TB Gold Plus; TB, tuberculosis. 

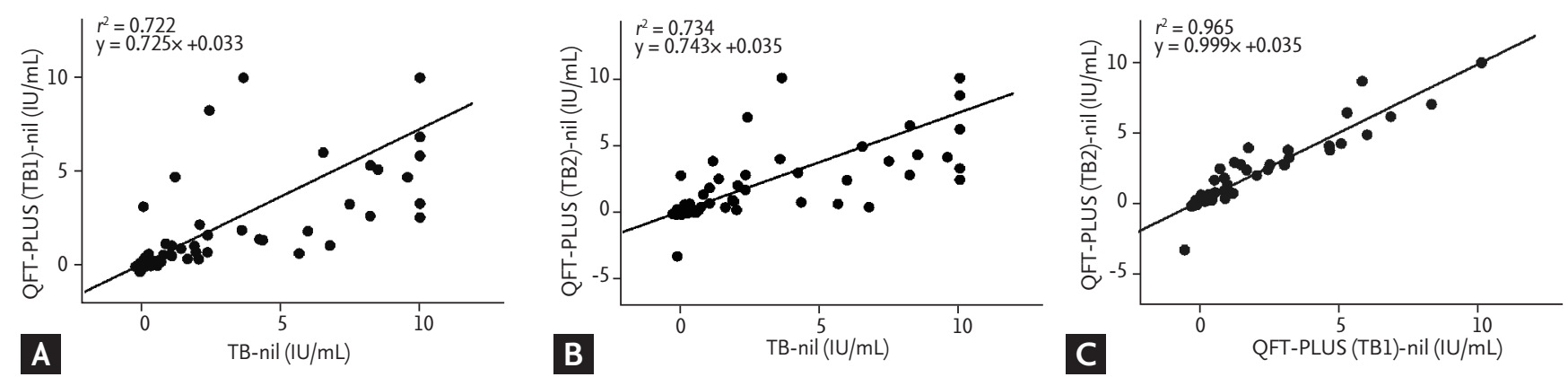

Figure 2. Linear regression plot of interferon- $\gamma$ concentration in (A) tuberculosis (TB) antigen-nil vs. TB1-nil, (B) TB antigennil vs. TB2-nil, and (C) TB1-nil vs. TB2-nil. QFT-PLUS, QuantiFERON-TB Gold Plus.

Table 3. Discordant results of QFT-GIT versus QFT-PLUS (n = 12)

\begin{tabular}{|c|c|c|c|c|c|c|}
\hline \multirow{3}{*}{ QFT-GIT results } & \multirow{3}{*}{ Immunosuppression } & \multirow{3}{*}{ TB infection risk } & \multirow{3}{*}{ Sample No. } & \multicolumn{3}{|c|}{ IFN- $\gamma, \mathrm{IU} / \mathrm{mL}$} \\
\hline & & & & \multirow{2}{*}{$\frac{\text { QFT-GIT }}{\text { TB-nil }}$} & \multicolumn{2}{|c|}{ QFT-PLUS } \\
\hline & & & & & TB1-nil & TB2-nil \\
\hline \multirow[t]{5}{*}{ Positive } & Yes & Biologic therapy & S8 & 0.38 & 0.06 & 0.04 \\
\hline & & & $\mathrm{S}_{5} 6$ & 0.65 & 0.03 & 0.06 \\
\hline & & & $\mathrm{S} 98$ & 0.69 & 0.12 & 0.02 \\
\hline & & & S117 & 0.77 & 0.21 & 0.22 \\
\hline & No & TB contact & S68 & 0.37 & 0.01 & 0.04 \\
\hline \multirow[t]{4}{*}{ Negative } & Yes & TB contact & $\mathrm{S} 18$ & 0.19 & 0.42 & 0.25 \\
\hline & & Biologic therapy & S147 & 0.15 & 3.15 & 2.8 \\
\hline & No & Others & $\mathrm{S}_{72}$ & 0.28 & 0.55 & 0.66 \\
\hline & & & S141 & 0.34 & 0.63 & 0.34 \\
\hline \multirow[t]{3}{*}{ Indeterminate } & No & Biologic therapy & $\mathrm{S} 26$ & -0.02 & o & 0.01 \\
\hline & & & $\mathrm{S}_{155}$ & 0.41 & $\mathrm{O}$ & 0.01 \\
\hline & & Others & $\mathrm{S}_{43}$ & 0 & 0 & 0 \\
\hline
\end{tabular}

QFT-GIT, QuantiFERON-TB Gold In-Tube; QFT-PLUS, QuantiFERON-TB Gold Plus; TB, tuberculosis; IFN- $\gamma$, interferon- $\gamma$.

к of 0.711 (Table 4$)$.

The median IFN- $\gamma$ release of TB $(3.01 \mathrm{IU} / \mathrm{mL}$; IQR, 1.80 to 10.00) did not differ significantly from that of TB1 (4.93 IU/mL; IQR, 1.15 to $10.00 ; p=1.000)$ and TB2 (4.17 $\mathrm{IU} / \mathrm{mL}$; IQR, 1.28 to $10.00 ; p=0.866)$ in the QFT-GITand QFT-PLUS-positive results $(\mathrm{n}=10)$. There were significant differences in the median IFN- $\gamma$ release of TB (0.01 IU/mL; IQR, 0.00 to 0.05) compared to that of TB1 (o.01 IU/mL; IQR, -0.01 to $0.02 ; p=0.007$ ) and TB2 (o IU/mL; IQR, -0.01 to $0.02 ; p=0.006$ ) in QFT-GIT- and QFT-PLUS-negative results $(n=48)$. ICC showed a good correlation between QFT-GIT and QFT-PLUS (TB1: ICC $=0.947, p<0.001 ;$ TB2: ICC = 0.951, $p<0.001)$.
There were six cases of discordant results. A statistical analysis could not be performed due to the small number of subjects. The IFN- $\gamma$ releases of the four QFTGIT samples were in the borderline range of 0.25 to 0.8 $\mathrm{IU} / \mathrm{mL}[25]$.

\section{Evaluation of the role of CD8+ T-cells}

The contribution of CD8+ T-cells was evaluated by investigating the difference in IFN- $\gamma$ release between TB2 and TB1 within the QFT-PLUS-positive group (Table 5). Nine subjects showed a TB2 minus TB1 value $>0.6$ $\mathrm{IU} / \mathrm{mL}$. Among them, active TB was the most frequent (n $=5)$, followed by other disease $(n=3)$ and LTBI screening 
Table 4. Results of QFT-GIT versus QFT-PLUS in 64 immunocompromised subjects

\begin{tabular}{|c|c|c|c|c|c|}
\hline \multirow{3}{*}{ QFT-GIT results } & \multirow{3}{*}{ No. of subjects } & \multicolumn{4}{|c|}{ QFT-PLUS results } \\
\hline & & \multicolumn{3}{|c|}{ Positive } & \multirow{2}{*}{ Negative } \\
\hline & & TB1-nil or TB2-nil & TB1-nil & TB2-nil & \\
\hline Negative & 50 & 2 & 2 & 1 & 48 \\
\hline Positive & 14 & 10 & 10 & 9 & 4 \\
\hline Total & 64 & 12 & 12 & 10 & 52 \\
\hline
\end{tabular}

QFT-GIT, QuantiFERON-TB Gold In-Tube; QFT-PLUS, QuantiFERON-TB Gold Plus; TB, tuberculosis.

Table 5. Difference in IFN- $\gamma$ level between TB2 and TB1 ([TB2-TB1] $>0.6$ IU/mL) based on TB infection risk in subjects with QFT-PLUS-positive results $(n=44)$

\begin{tabular}{lccc}
\hline TB2-TB1, IU/mL & Active TB/TB contact & Biologic therapy/other diseases & Total \\
\hline$>0.6$ & 5 & 4 & 9 \\
$\leq 0.6$ & 14 & 21 & 35 \\
Total & 19 & 25 & 44 \\
\hline
\end{tabular}

IFN- $\gamma$, interferon- $\gamma$; TB, tuberculosis; QFT-PLUS, QuantiFERON-TB Gold Plus.

before biologic therapy $(n=1)$. There was no significant difference in the proportion of a TB2 minus TB1 IFN- $\gamma$ release $>0.6 \mathrm{IU} / \mathrm{mL}$ among the four groups $(p=0.133)$. This was also seen between the high probability of recent infection group (active TB and TB contact: $\mathrm{n}=5 / 19$, $26.3 \%$ ) and low probability of infection group (biologic therapy and other disease: $n=4 / 25,16.0 \%)(p=0.401$ ). Furthermore, there was no significant difference in the median value of TB2 minus T'B1 within QFT-PLUSpositive subjects among the four groups $(p=0.169)$ or between the two groups mentioned above $(p=0.147)$.

\section{Comparison of TB1 and TB2 of the QFT-PLUS assay}

The $\mathrm{TB}_{1}$ and $\mathrm{TB} 2$ results were compatible, resulting in an agreement of $97.1 \%$ and a Cohen's $\kappa$ of 0.931 .

The median IFN- $\gamma$ release of TB2 $(2.83 \mathrm{IU} / \mathrm{mL}$; IQR, 0.84 to 6.93 ) did not differ significantly from that of TB1 (2.39 IU/mL; IQR, 0.78 to 6.64) in the 44 QFT-PLUS positive results $(p=0.422)$. TB2 $(0.01 \mathrm{IU} / \mathrm{mL} ; \mathrm{IQR},-0.01$ to ○.03) did not differ significantly from TB1 (o.01 IU/mL; IQR, -0.05 to 0.03 ) in 93 the QFT-PLUS negative results $(p=0.665)$. The ICC between TB1 and TB2 was 0.991 ( $p<$ $0.001)$, which suggested good correlation. The linear regression analyses also showed good correlation between TB1 and TB2 $\left(r^{2}=0.965\right)$ (Fig. $\left.2 \mathrm{C}\right)$.

There were four cases of discordant results (one of
TB1-negative and TB2-positive, three of TB1-positive and TB1-negative). A statistical analysis could not be performed due to the small number of subjects.

\section{DISCUSSION}

Most previous studies comparing the performances of the QFT-PLUS and QFT-GIT tests were performed in European countries with a low TB burden and showed comparable results for diagnosing TB infection $[19,21,22,26]$. Our study provides further evidence that the QFT-PLUS and QFT-GIT are highly comparable based on the results of agreement, Cohen's $\kappa$, and ICC between the tests even in a TB endemic country. The test results did not differ significantly by immune status or TB infection risk. This indicates the usefulness of QFT-PLUS compared to the QFT-GIT even in an intermediate T'B burden country like Korea.

We demonstrated here that the QFT-PLUS results were highly comparable to those of the QFT-GIT in immunocompromised subjects as well. Previous studies revealed that QFT-PLUS showed higher sensitivity in HIV and TB co-infected subjects than the QFT-GIT $[20,27]$. In this study, the majority of subjects had been assigned to an LTBI screening before biologic therapy 
group and had IMIDs ( $\mathrm{n}=85 / 137,62.0 \%)$. Among these patients, the majority were immunocompromised ( $\mathrm{n}=$ $53 / 85,62.4 \%)$, due to the high usage of disease-modifying anti-rheumatic drugs or steroids. Anti-tumor necrosis factor- $\alpha$ antagonist or other biologics are being increasingly used in such patients [28]. As a result, this might lead to an increased risk of active TB, which mostly results from the reactivation of LTBI in an intermediate TB endemic country $[28,29]$. Our study findings suggested that QFT-PLUS could be interchangeably used with QFT-GIT to diagnose TB infection in patients with immunocompromised status (especially those resulting from $\mathrm{CD}_{4}+\mathrm{T}$-cell impairments) for detection of LTBI, in addition to a recent study by Ryu et al. [30]. Our study indicated that the positive rate of QFT-GIT ( $\mathrm{n}=14 / 64$, 21.9\%) was slightly higher than that of QFT-PLUS (n $=12 / 64,18.8 \%$ ) in immunocompromised hosts, which could be attributed to high variability of the cut-off value of QFT-GIT of $\pm 0.6 \mathrm{IU} / \mathrm{mL}$, resulting in false-positive results and conversion/reversion events [25,31]. All four QFT-GIT-positive and QFT-PLUS-negative cases showed IFN- $\gamma$ level in the range of 0.38 to $0.77 \mathrm{IU} / \mathrm{mL}$. Further studies to compare between QFT-GIT and QFT-PLUS in immunocompromised subjects including larger cohort-size and long-term clinical follow-up are needed.

A previous study showed that CD8+ T-cells were frequently detected in individuals with active TB compared to those with LTBI, which correlates to a high TB burden [16]. Furthermore, other studies showed that higher IFN- $\gamma$ release in TB2 compared with T'B1 was associated with recent TB infection [19,22]. The high-risk TB infection group has a greater chance of recent infection than the low-risk group. However, in our study, there was no significant difference in the proportion of TB2 antigen response (TB2-TB1 difference of > $0.6 \mathrm{IU} /$ $\mathrm{mL}$ ) between the two groups, nor was there a significant difference in the median IFN- $\gamma$ release of TB2-TB1 between the groups. This finding contradicts that of a previous study of the Netherlands and Belgium [19], and could be explained as follows. First, QFT-PLUS as well as QFT-GIT, doesn't have capability to distinguish between recent TB infection and remote TB infection. Second, while QFT-PLUS could distinguish between recent TB infection and remote TB infection, it failed to show greater TB2-antigen response in the high-risk group in our study, which may be due to the low number of study participants. Furthermore, since South Korea is a TB-prevalent country, there is possibility of unnoticed, hidden infection even in the low-risk TB infection group. Further studies are needed to reveal the role of the CD8+ T-cell-response component of QFT-PLUS test in the diagnoses of TB infection.

The positive rate of either test was higher in the other disease group (62.5\%) than in the TB contact group $(42.9 \%, p=0.240)$. This is contradictory with the fact that the TB contact population usually has a higher possibility of LTBI infection than the general population. This phenomenon may be attributed to the relatively low number of participants in TB contact group, and in part, to the slightly lower median-participant age in TB contact group (53.93; IQR, 45.75 to 62.25) versus other disease group (57.0; IQR, 49.25 to 64.75), despite absence of statistically significant difference [1].

In this study, the positive IFN- $\gamma$ release level was significantly higher on the QFT-GIT than the TB1 on the QFT-PLUS. This might be attributable to the presence of the peptide antigen TB 7.7 in the QFT-GIT kit, which was removed from the QFT-PLUS kit [18]. Our study also showed that TB2 induced significantly lower IFN- $\gamma$ release levels compared to that of TB in the QFT-GIT and QFT-PLUS positive results, which is in contrast with the previous study [19]. We expected a higher response of TB2 than TB based on the fact that TB2 contains modified peptides that elicit a CD8+ T-cell reaction in addition to the $\mathrm{CD}_{4}+\mathrm{T}$-cell response [18]. This unexpected result might have been due to the low number of patients with a recent TB infection in this study group.

The current diagnostic standard of IGRA tests emphasizes the importance of dichotomous value, positive or negative, over that of absolute IFN- $\gamma$ level. However, based on findings of a recent study of positive association between higher absolute IFN- $\gamma$ level and TB disease occurrence, absolute IFN- $\gamma$ level may be considered as additional important information that enables diagnosis of TB infection [32]; hence, in our study, comparison data of absolute IFN- $\gamma$ levels were presented even though they comprised negative values with similar median and IQR values between the two groups.

The discordant IFN- $\gamma$ release between the QFT-GIT and the QFT-PLUS was mostly in the borderline range ( $\mathrm{n}=8 / 12,66.7 \%$ ). This indicates the possibility of test 
variability instead of true inter-test differences [25]. Among the 12 cases of discordant results, five were QFTGIT-positive and QFT-PLUS-negative. This might be due to the relatively low IFN $-\gamma$ release of TB1 compared to the QFT-GIT or the low number of patients with a recent TB infection as mentioned above.

Our study has some limitations. First, our sample size was relatively small, especially in the high-risk TB infection groups. This might have influenced the statistical results of the true IFN- $\gamma$ release difference (TB2-TB1) comparison between TB infection risk groups, thereby causing confusion in the interpretation of the role of CD8+ T-cells as a predictor for TB burden or recent TB infection. Second, we could not evaluate the sensitivity and specificity of the QFT-PLUS because there is still no gold standard test for LTBI infection. Thus, we could not evaluate the actual accuracy of the QFT-PLUS or properly assess the discordant results between the tests. Third, this study was processed in Korea, a TB-prevalent country. This might have raised the problem of including a relatively high number of recent LTBI-infected subjects even in the low-risk TB infection group than expected. Fourth, this study included a relatively broad range of immunocompromised statuses. We could have drawn more meaningful results from the QFT-PLUS if we included only large numbers of $\mathrm{CD}_{4}+$-deficient subjects since the QFT-PLUS has the additional ability of inducing the CD8+ T-cell response.

In conclusion, our study showed highly comparable results between the QFT-PLUS and QFT-GIT in Korea, even in immunocompromised subjects, which suggests that QFT-PLUS has potential for use as an alternate modality to QFT-GIT in the diagnosis of TB infection. However, this study could not provide supporting evidence for the use of the QFT-PLUS in distinguishing between recent TB infection (such as active TB or recent LTBI) and remote TB infection, which might be due to the low numbers of subjects and intermediate TB burden in Korea.

\section{KEY MESSAGE}

1. QuantiFERON-TB Gold PLUS (QFT-PLUS) showed highly comparable results to those of QuantiFERON-TB Gold In-Tube (QFT-GIT) for diagnosing tuberculosis (TB) infection in Korea, as well as in immunocompromised subjects.

2. QFT-PLUS showed no significant difference in the proportion of TB2 minus TB1-interferon- $\gamma$ release of $>0.6 \mathrm{IU} / \mathrm{mL}$ between the highrisk TB infection group and the low-risk TB infection group, which might be due to low numbers of subjects and the intermediate TB burden in Korea.

\section{Conflict of interest}

No potential conflict of interest relevant to this article was reported.

\section{REFERENCES}

1. World Health Organization. Global Tuberculosis Report 2017. Geneva (CH): World Health Organization, 2017.

2. Getahun H, Matteelli A, Chaisson RE, Raviglione M. Latent Mycobacterium tuberculosis infection. N Engl J Med 2015;372:2127-2135.

3. Trauer JM, Moyo N, Tay EL, et al. Risk of active tuberculosis in the five years following infection . . 15\%? Chest 2016;149:516-525.

4. Jasmer RM, Nahid P, Hopewell PC. Clinical practice. Latent tuberculosis infection. N Engl J Med 2002;347:18601866.

5. Lonnroth K, Migliori GB, Abubakar I, et al. Towards tuberculosis elimination: an action framework for low-incidence countries. Eur Respir J 2015;45:928-952.

6. Goletti D, Sanduzzi A, Delogu G. Performance of the tuberculin skin test and interferon- $\gamma$ release assays: an update on the accuracy, cutoff stratification, and new potential immune-based approaches. J Rheumatol Suppl 2014;91:24-31.

7. van Ingen J, de Zwaan R, Dekhuijzen R, Boeree M, van Soolingen D. Region of difference 1 in nontuberculous Mycobacterium species adds a phylogenetic and taxonomical character. J Bacteriol 2009;191:5865-5867.

8. Santin M, Munoz L, Rigau D. Interferon- $\gamma$ release assays for the diagnosis of tuberculosis and tuberculosis infection in HIV-infected adults: a systematic review and meta-analysis. PLoS One 2012;7:e32482. 
9. Mandalakas AM, Detjen AK, Hesseling AC, Benedetti A, Menzies D. Interferon-gamma release assays and childhood tuberculosis: systematic review and meta-analysis. Int J Tuberc Lung Dis 2011;15:1018-1032.

10. Rangaka MX, Wilkinson KA, Glynn JR, et al. Predictive value of interferon- $\gamma$ release assays for incident active tuberculosis: a systematic review and meta-analysis. Lancet Infect Dis 2012;12:45-55.

11. Brighenti $\mathrm{S}$, Andersson J. Induction and regulation of CD8+ cytolytic T cells in human tuberculosis and HIV infection. Biochem Biophys Res Commun 2010;396:50-57.

12. Cooper AM. Cell-mediated immune responses in tuberculosis. Annu Rev Immunol 2009;27:393-422.

13. Bruns H, Meinken C, Schauenberg P, et al. Anti-TNF immunotherapy reduces CD8+ T cell-mediated antimicrobial activity against Mycobacterium tuberculosis in humans. J Clin Invest 2009;119:1167-1177.

14. Mazzaccaro RJ, Stenger S, Rock KL, et al. Cytotoxic T lymphocytes in resistance to tuberculosis. Adv Exp Med Biol 1998;452:85-101.

15. Lewinsohn DA, Heinzel AS, Gardner JM, Zhu L, Alderson MR, Lewinsohn DM. Mycobacterium tuberculosis-specific CD8+ T cells preferentially recognize heavily infected cells. Am J Respir Crit Care Med 2003;168:1346-1152.

16. Rozot V, Vigano S, Mazza-Stalder J, et al. Mycobacterium tuberculosis-specific CD8+ T cells are functionally and phenotypically different between latent infection and active disease. Eur J Immunol 2013;43:1568-1577.

17. Petruccioli E, Chiacchio T, Pepponi I, et al. First characterization of the $\mathrm{CD}_{4}$ and CD8 T-cell responses to QuantiFERON-TB Plus. J Infect 2016;73:588-597.

18. QIAGEN. QuantiFERON-TB Gold Plus (QFT-PLUS), ELISA Package Insert [Internet]. Venlo (NL): QIAGEN, 2015 [cited 2019 Sep 30]. Available from: https://www.qiagen. com.

19. Pieterman ED, Liqui Lung FG, Verbon A, et al. A multicentre verification study of the QuantiFERON(®)-TB Gold Plus assay. Tuberculosis 2018;108:136-142.

20. Petruccioli E, Vanini V, Chiacchio T, et al. Analytical evaluation of QuantiFERON-Plus and QuantiFERON-Gold In-tube assays in subjects with or without tuberculosis. Tuberculosis 2017;106:38-43.

21. Hoffmann H, Avsar K, Gores R, Mavi SC, Hofmann-Thiel S. Equal sensitivity of the new generation QuantiFER-
ON-TB Gold plus in direct comparison with the previous test version QuantiFERON-TB Gold IT. Clin Microbiol Infect 2016;22:701-703.

22. Barcellini L, Borroni E, Brown J, et al. First evaluation of QuantiFERON-TB Gold Plus performance in contact screening. Eur Respir J 2016;48:1411-1419.

23. Korea Centers for Disease Control and Prevention. Korean Guidelines for Tuberculosis. 3rd ed. Seoul (KR): Korea Centers for Disease Control and Prevention, 2017.

24. Blumberg HM, Burman WJ, Chaisson RE, et al. American Thoracic Society/Centers for Disease Control and Prevention/Infectious Diseases Society of America: treatment of tuberculosis. Am J Respir Crit Care Med 2003;167:603662.

25. Metcalfe JZ, Cattamanchi A, McCulloch CE, Lew JD, Ha NP, Graviss EA. Test variability of the QuantiFERON-TB gold in-tube assay in clinical practice. Am J Respir Crit Care Med 2013;187:206-211.

26. Yi L, Sasaki Y, Nagai H, et al. Evaluation of QuantiFERON-TB Gold Plus for detection of Mycobacterium tuberculosis infection in Japan. Sci Rep 2016;6:30617.

27. Telisinghe L, Amofa-Sekyi M, Maluzi K, et al. The sensitivity of the QuantiFERON(®)-TB Gold Plus assay in Zambian adults with active tuberculosis. Int J Tuberc Lung Dis 2017;21:690-696.

28. Keane J, Gershon S, Wise RP, et al. Tuberculosis associated with infliximab, a tumor necrosis factor alpha-neutralizing agent. N Engl J Med 2001;345:1098-1104.

29. Solovic I, Sester M, Gomez-Reino JJ, et al. The risk of tuberculosis related to tumour necrosis factor antagonist therapies: a TBNET consensus statement. Eur Respir J 2010;36:1185-1206.

30. Ryu MR, Park MS, Cho EH, et al. Comparative evaluation of QuantiFERON-TB Gold In-Tube and QuantiFERON-TB Gold Plus in diagnosis of latent tuberculosis infection in immunocompromised patients. J Clin Microbiol 2018;56:e00438-18.

31. Banaei N, Gaur RL, Pai M. Interferon gamma release assays for latent tuberculosis: what are the sources of variability? J Clin Microbiol 2016;54:845-850.

32. Andrews JR, Nemes E, Tameris M, et al. Serial QuantiFERON testing and tuberculosis disease risk among young children: an observational cohort study. Lancet Respir Med 2017;5:282-290. 